\title{
Urban Partnerships to Address Health Literacy in High Need Populations
}

\author{
Abiola O. Keller, Amy Vuyk, and Joshua Knox
}

\begin{abstract}
Low health literacy disproportionately affects racial and ethnic minority communities and lowerincome socioeconomic groups. To address this critical determinant of health inequity, two nonprofit organizations, Repairers of the Breach, a day shelter for individuals experiencing homelessness, and Bread of Healing a neighborhood-based clinic serving individuals with low incomes, partnered with researchers at Marquette University to implement and evaluate an evidence-supported health literacy program. The partnership delivered the curriculum in seven one-hour sessions over seven weeks. The program attendees were predominantly African American men and women from 19-73 years old. Most participants had formal education ranging from elementary school to some college. Forty individuals attended at least one class and 14 attendees completed 4 or more classes. Program completers demonstrated gains in confidence and topic knowledge. Most interviewees reported a personal/family need for the program, acceptability of the group format, and the ability to learn the skills they needed for self-care. The project used a successful collaboration between community-based organizations serving vulnerable populations and an urban academic institution to demonstrate the necessity, feasibility, acceptability, and effectiveness of formal health literacy education in adults with low incomes or who are experiencing homelessness. Urban and metropolitan serving institutions can work in partnership with community to address low health literacy in high need populations.
\end{abstract}

Keywords: HEAL program; health education; community-academic collaboration

\section{Introduction}

To promote health, improve health outcomes, and achieve equity, individuals must be able to understand and use the health information they hear, read, and see from numerous sources. Yet, approximately 80 million adults in the U.S. have difficulty using everyday health information (Kutner, Greenberg, Jin, \& Paulsen, 2006). Health literacy, defined as the degree to which individuals have the capacity to obtain, process, and understand basic health information and services need to make appropriate health decisions (Ratzan \& Parker, 2000) is an important determinant of health. Research has shown low health literacy to be associated with adverse health outcomes (Berkman, Sheridan, Donahue, Halpern, \& Crotty, 2011) and poorer use of health care services (Griffey, Kennedy, D'Agostino McGowan, Goodman, \& Kaphingst, 2014). While low health literacy affects people of all races, ages, education, and income levels, rates are higher among minority and lower socioeconomic groups (Kutner et al., 2006). Given the disproportionate burden of low health literacy and its adverse impact on health, improving health literacy had been recognized as a critical mechanism for improving health and health outcomes (U.S. Department of Health and Human Services, 2010). Notably, improving health literacy is included as one of the Healthy People 2020 objectives (Office of Disease Prevention and Health Promotion, 2019). 
Institutions of higher education can play a major role in addressing inequities in determinants of health such as health literacy. In addition to collaborating with community to deliver healthpromoting services and programs, urban and metropolitan serving institutions may also serve as evaluation partners. Collecting and analyzing data to determine the feasibility and effectiveness of community-based programs in order to better understand what does and does not work is critical for optimizing program outcomes and maximizing the return on investment. (Centers for Disease Control and Prevention, 2019). In this article, we present an example of leveraging a community-academic partnership to deliver and evaluate a health literacy intervention in a high need population.

In response to a community-driven agenda to elevate the health and well-being of community residents (Milwaukee Health Department) and in recognition of the potential for limited health literacy to reinforce existing inequalities, researchers at Marquette University, an urban institution, partnered with two local clinics serving individuals with low incomes or who are experiencing homelessness to address low health literacy. Available evidence suggests that health literacy can be increased through focused interventions (Taggart et al., 2012). Moreover, interventions delivered by non-physicians (Dennis et al., 2012), or in a community setting (Taggart et al., 2012) are promising approaches. The Health Education and Literacy (HEAL) Program (Literacy for Life, 2016) is a community-based, health literacy course. Although the HEAL Program has been shown to increase participants' confidence and health knowledge (Mellor \& Uselton, 2016), this has not been investigated in lower resourced settings. Moreover, participants' perceptions of the necessity, acceptability, feasibility, and safety of the intervention have not been examined. Information on these parameters is essential for guiding efforts to augment health literacy skills in underserved populations. To address this gap, a community-academic partnership sought to assess the necessity, acceptability, feasibility, fidelity, safety, and effectiveness of the HEAL program in an urban setting.

\section{Methods}

\section{Design}

This qualitative and quantitative study explored the six critical parameters - necessity, acceptability, feasibility, safety, fidelity, and effectiveness - of a health literacy intervention in an urban community. This approach has been used in previous intervention research with people facing multiple barriers (Bekhet, Zauszniewski, \& Matel-Anderson, 2012). Data were collected from participants during face-to-face interviews at the conclusion of the intervention. This study was approved as minimal risk by the Marquette University Institutional Review Board.

Setting

Repairers of the Breach (ROB). Repairers of the Breach is a nonprofit day shelter for individuals experiencing homelessness. Repairers is engaged in healing activities that build community awareness and pave the way for individual improvement through nutrition, fellowship, counseling and support groups, violence reduction, substance abuse recovery, leadership, and 
volunteerism. Repairers also operates a free medical clinic serving individuals experiencing homelessness and the local community.

Bread of Healing $(\mathrm{BOH})$. Bread of Healing is a nonprofit neighborhood-based clinic serving individuals with low incomes. Bread of Healing provides medical, pharmacy, dental, counseling, social service, and health education services.

\section{Participants}

For the study, the study team recruited a convenience sample of adults receiving medical services at $\mathrm{ROB}$ or $\mathrm{BOH}$ medical clinics. Individuals were eligible for the study if they attended ROB or $\mathrm{BOH}$ medical clinics, were willing to attend up to 8 intervention sessions, and able to provide information via pre and post surveys and an interview.

\section{Procedure}

The community partners informed recruitment approaches for the study. At ROB, researchers distributed printed materials about the study and posted flyers in prominent places. In addition, the investigators had one-on-one conversations with prospective participants in the clinic. At $\mathrm{BOH}$, investigators held an information study where they presented general information about the study. At both sites, anyone who was interested in participating and eligible was invited to attend the first intervention session.

\section{The Intervention}

Literacy for Life (2016), an independent non-profit organization of the William and Mary School of Education in Williamsburg, VA, developed and disseminates the intervention used here, entitled Health Education and Literacy (HEAL) Program. Prior to beginning the study, the authors traveled to the Literacy for Life Learning Center and completed the 1-day certification training course necessary to obtain a license for administering the HEAL program. The research team conducted the HEAL program twice, once at ROB and once at BOH. Consistent with the established protocol, the HEAL program sessions took place in a small group format. Each session was held in a private classroom. Dr. Keller and Mr. Knox alternated teaching the sessions at BOH. Ms. Vuyk taught the curriculum at ROB with support from Mr. Knox. The research team delivered the HEAL program curriculum in seven one-hour sessions over seven weeks. They addressed topics such as how to describe symptoms to a doctor, reading and understanding medication instructions, understanding when to use non-emergent health care services versus using the emergency room, understanding medical forms, and identifying healthy lifestyle choices. Each of the sessions was conducted by one or two members of the research team. 
Measures and Instruments

Measuring and Evaluating the Intervention Parameters. The team assessed the necessity of the HEAL program by asking participants if they felt they needed the program and if they thought that their friends and family would benefit. The authors also gauged necessity using the participants' baseline scores on the Rapid Estimate of Adult Literacy in Medicine-Short Form (REALM-SF), a 7-item word recognition test. The REALM-SF has been validated, and fieldtested in diverse research settings (Arozullah et al., 2007). Possible scores on the REALM-SF range from 0 to 7 . Individuals with a score of zero (grade equivalent $=$ third grade or below) will be unable to read most low-literacy materials, those with a score of one to three (grade equivalent $=$ fourth to sixth grade) will need low-literacy materials and be likely unable to read prescription labels. Adults with a REALM-SF score of four to six (grade equivalent = seventh to eighth grade) often struggle with most patient-education materials and would benefit from lowliteracy materials. Those with a score of seven (grade equivalent $=$ high school) will likely be able to read most patient education materials. Additionally, this study examined baseline scores on a brief questionnaire assessing participants' confidence for and knowledge of the topics covered in the HEAL program. Confidence was measured by asking how comfortable and confident participants felt in doing routine health tasks such as asking providers questions, competing forms, and determining when and where to seek care. Available responses for each item were on a 5-point Likert scale including not at all, not very, somewhat, very, and extremely with higher numbers indicating more confidence. The authors assessed the knowledge gained with eight true or false questions based on the program curriculum.

To evaluate acceptability of the HEAL program, participants were asked to describe what part or parts of the program were most, and least interesting. They were also asked whether they thought the program content and the group format were appropriate for them.

The feasibility of the program was assessed by asking participants to describe what part or parts of the program were easiest and most challenging, and whether they thought the number of and length of each session was appropriate for them.

To determine program fidelity, participants were asked if they thought they learned the skills they needed to take care of their health and what would have helped them to learn better. Fidelity was also assessed by administering the brief questionnaire to assess participants' confidence for and knowledge of the topics covered in the HEAL program at the completion of the curriculum. To compare the pre and post confidence and knowledge scores a paired Wilcoxon signed-rank test was performed. The Wilcoxon signed-rank test is similar to the paired t-test but is more suitable when the data are non-normally distributed (McDonald, 2014). The research team hypothesized that the post-program scores would increase if the program content was taught effectively.

The safety of the HEAL program was measured by asking participants to describe the part or parts of the program that were the most, and least, uncomfortable or distressing and whether they had any worries about confidentiality during the group sessions.

The effectiveness of the HEAL program was evaluated by asking participants to describe the part or parts of the program that were the most, and least, helpful in teaching them about taking care 
of their health. Participants were also asked for feedback on ways that the program could be improved.

All participants were asked to provide demographic information (age, gender, race, ethnicity, and education). Perceived health status was evaluated using a single item question asking participants to rate their general health on a scale from 1 to 5 with 1 being poor and 5 being excellent.

\section{Results}

The Study Sample

Forty adults attended at least one HEAL program session $(\mathrm{ROB}=24, \mathrm{BOH}=16)$ with 13 attending four or more sessions $(\mathrm{ROB}=7, \mathrm{BOH}=6)$. Fourteen adults completed the postprogram evaluation $(\mathrm{ROB}=9, \mathrm{BOH}=5)$. Thirteen of the 14 adults identified as African American. Among those who completed the post-program evaluation, 7 were women (50\%) and 7 men (50\%). Participants in the program evaluation were between the ages of 27 and 64 years (mean, 50), the majority had a high school education or less $(\mathrm{n}=12,86 \%)$ and rated their health to be good, very good, or excellent $(n=11,79 \%)$.

\section{Evaluation of Intervention Parameters}

Necessity. The baseline REALM-SF scores, as well as participants' confidence for and knowledge of the program topics, were assessed as indicators of the level of need for the HEAL program. The REALM-SF scores of the study sample ranged from one to seven (mean, 4.6), baseline confidence scores ranged from three to five (mean, 3.9), baseline knowledge were between zero and six (mean, 4.1). Most program completers reported feeling that they needed the program $(86 \%)$ or that their family and friends needed the program $(79 \%)$. One participant said, "At first I didn't [feel I needed the program], but now that I went through it, I learned a lot of stuff. It was beneficial." Another shared, "I can say I need it. Even though I knew some of it, it helped freshen my mind."

Acceptability. When asked what part or parts of the program was most interesting, the most frequently discussed topics included communicating with providers $(n=4)$ and diet and nutrition $(n=4)$. One participant said, "Health wise about the weight, learning how much calories in soda." Information about smoking cessation and medication taking emerged as the least interesting. With regards to smoking cessation, one participant explained by saying "Smoking, not trying to quit right now." When asked about the appropriateness of the group format, many participants responded positively. Responses included "It was fun cause you learn more from different people." and "It was awesome cause you learn something from everybody."

Feasibility. Half of the participants reported that nothing about the program was difficult. Among those who reported finding part of the program challenging, the most frequently $(n=3)$ discussed challenge had to do with making changes related to diet and nutrition. One participant described this by saying "Eating right. I am still in the process of eating right. Sometimes I eat too much cake." Another added, "I have to watch what I eat even though I want fast food, I can't have it." Six individuals responded that the content pertaining to diet and nutrition was the easiest. This 
was exemplified by comments such as "I used to eat a lot but [the class] told you different things to eat to watch your weight" and "I never knew to go to the store and look at labels. I used to go to the store and just pick up stuff." The majority of participants (64\%) wanted either more class time or longer sessions, "The hour was too short" or additional classes "for a beginning it was [long enough], but I wish they has a Step 2."

Fidelity. Approximately $93 \%$ of participants felt they were able to learn the skills they needed to take care of their health. One said, "Since I have been in the class I'm controlling my blood pressure better and eating better, less fried food, more baked food, more veggies." When asked about what would have helped them learn better, $36 \%$ said nothing more was needed, $29 \%$ commented on individual factors such as, "maybe if I wasn't so stubborn" or "pay more attention," and 14\% requested changes to the methods used to deliver the content, including writing on the board more and using a projector. Post-program confidence and knowledge scores were measured as an indicator of the fidelity of the program. After completing the program, confidence scores ranged from 2.7 to 5 (mean, 4.1). Using the Wilcoxon signed-rank test, there was no statistically significant difference between the pre and post confidence scores ( $p$-value $>$ 0.2 ). The post knowledge scores were overall higher than the pre-scores and ranged from 2 to 7 (mean, 5.9). Based on the Wilcoxon signed-rank test there was a statistically significant difference between the pre and post knowledge scores $(0.02<$ p-value $<0.05)$.

Safety. Over half (57\%) of the participants reported that nothing made them feel uncomfortable. Three participants reported discomfort with activities focused on behavior change. One participant described this by saying. "Trying to force you to eat something you don't know anything about, never heard of. I like soul food." Most adults (93\%) were not concerned about confidentiality during the group sessions and felt they were able to control the information that was shared. This was exemplified by statements such as, "if I want them to know something, I'll tell them."

Effectiveness. When asked what part or parts of the program were the most helpful in teaching them about taking care of their health the majority (79\%) of participants reported that everything was helpful. The most frequently mentioned topic was learning about communicating with providers and preparing for appointments. With regards to ways the program could be improved, responses focused on participants' desire for more program sessions.

\section{Discussion}

This is the first study to evaluate the necessity, acceptability, feasibility, safety, fidelity, and effectiveness of the HEAL program in individuals with low incomes or who are experiencing homelessness. Regarding fidelity and effectiveness, participants' knowledge scores were significantly higher at the end of the program compared to their scores before the program. Moreover, most participants reported that everything they learned was helpful in helping them take care of their health.

The study findings indicate that there is a great need for health literacy training in populations with low resources. Given that the study participants were primarily (93\%) African American, the findings are consistent with previous research suggesting that racial and ethnic minorities are 
at increased risk for having low health literacy (Kutner et al., 2006). In addition to demonstrating need, this study also found evidence that adults at the two clinics desired health literacy training and most notably, they found the topics included in the HEAL program acceptable and wanted more training sessions.

Delivering health literacy training programs in a group format within a community setting may be a valuable method for increasing access to these programs. This study showed that learning about and discussing health topics in a group format did not cause participants discomfort and in fact, participants valued being able to share their experiences and listen to others.

Throughout this community-academic partnership to address health literacy, the research team overcame challenges and learned valuable lessons. These may be useful to other urban and metropolitan universities seeking to influence health outcomes in their communities. A key challenge for the study team was participant attrition. It was encouraging that forty total participants attended at least one class. However, only 14 participants attended most of the classes and completed the post-assessments. The high attrition rate possibly resulted from the forced transience of the population, the degree of uncertainty in their daily schedules, personal or family illness, and other competing commitments such as job or housing searches. In addition to securing the support of organizational leaders, the successful implementation of group-based, multiple session programs, like the HEAL program, requires identifying champions for the classes amongst the organizational staff and program participants. Other critical components for success include advertising the classes beforehand in the clinics and securing dedicated space within the community partners' facilities for the classes.

While healthy food was provided at each session, a modest financial incentive was provided only at the first and last session. Financial incentives played a larger role in attendance at ROB with a more significant drop in attendance after the incentives diminished. It is recommended that future classes provide healthy food and modest financial incentives or small non-monetary items at each session to encourage attendance if utilizing this course with individuals with low incomes or who are experiencing homelessness.

Another challenge encountered was developing class cohesion. At both sites, the degree of engagement and enthusiasm for the curriculum was remarkable; however, a notably higher level of class cohesion and belonging occurred among ROB participants. Additionally, the research team noted robust discussions about changing health behaviors at ROB. There were discussions of the same issues at $\mathrm{BOH}$ but less participant commitment to behavior change. Speculatively, the different cohort constituencies, low income vs. experiencing homelessness, may explain this observation. Never the less a group dynamic that is cooperative and encourages respect of individual differences and abilities is essential for promoting learning. As such, it is recommended that instructors actively build class cohesion and model healthy behaviors for future classes.

The qualifications and experience of the HEAL teachers have implications for both this study and future interventions. All members of this research team were qualified adult educators with formal teacher training and certifications as health care professionals. Our qualifications as teachers were certainly an asset to the program. However, our background is unusual compared 
to most health literacy programming including typical HEAL courses where the instructors are either adult educators or healthcare professionals but not both. Further, we recognize our own limitations as teachers in this population. The students in the course were atypical compared to the graduate health professions students whom we ordinarily teach. Instructors planning to teach health literacy classes in low literacy populations need to be able to recognize basic literacy issues, adapt their teaching methods in response, and demonstrate flexibility and patience in classroom management of variations in attention, participation, and interruption. We suggest that others who are considering teaching health literacy take formal training such as the HEAL program provides or use online education through the Centers for Disease Control and Prevention (Centers for Disease Control and Prevention, 2018).

Future researchers and others working in this sphere need to consider both the sustainability and feasibility of community partnerships in health literacy programming prior to initiation. This study was externally grant funded by the nccPA Health Foundation Kathy J. Pedersen Grant to Promote Equitable Care and a matching donation from the Hepburn Foundation. The authors' institution, Marquette University, has continued to support licensing fees that have allowed the program to continue another year. Two of the authors, (Knox and Vuyk) have continued to teach the classes at the ROB site. A program to train physician-assistant students remotely to deliver the HEAL curriculum is currently in the planning stages. However, the authors recognize that factors including availability of funding, trustworthy relationships with community partners, and qualified available teaching staff may be serious impediments to others planning similar interventions.

\section{Conclusion}

In times of diminishing public resources and increasingly complex social problems, urban and metropolitan universities, and especially those universities with social missions, should be investigating and supporting collaborations with their communities and community-based organizations to curb urban decline in the areas of housing, violence, employment, education, and healthcare. This community-academic partnership to improve health literacy illustrates the value of fostering community-academic networks focused on addressing critical community issues and demonstrates the key role urban and metropolitan universities can play in improving the health of all groups in the cities they serve.

\section{Acknowledgments}

This research was supported in part by the Kathy J. Pedersen Grant to Promote Equitable Care supported by the nccPA Health Foundation. Additional support provided by the Hepburn Family. 


\section{References}

Arozullah, A. M., Yarnold, P. R., Bennett, C. L., Soltysik, R. C., Wolf, M. S., Ferreira, R. M., .. . Davis, T. (2007). Development and validation of a short-form, rapid estimate of adult literacy in medicine. Med Care, 45(11), 1026-1033. https://doi.org/10.1097/MLR.0b013e3180616c1b

Bekhet, A. K., Zauszniewski, J. A., \& Matel-Anderson, D. M. (2012). Resourcefulness training intervention: assessing critical parameters from relocated older adults' perspectives. Issues Ment Health Nurs, 33(7), 430-435. https://doi.org/10.3109/01612840.2012.664802

Berkman, N. D., Sheridan, S. L., Donahue, K. E., Halpern, D. J., \& Crotty, K. (2011). Low health literacy and health outcomes: an updated systematic review. Ann Intern Med, 155(2), $97-$ 107. https://doi.org/10.7326/0003-4819-155-2-201107190-00005

Centers for Disease Control and Prevention. (June 29, 2018). Health Literacy. Retrieved from https://www.cdc.gov/healthliteracy/gettraining.html

Centers for Disease Control and Prevention. (March 11, 2019). Program Performance and Evaluation Office (PPEO)-Program Evaluation. Retrieved from https://www.cdc.gov/eval/index.htm

Dennis, S., Williams, A., Taggart, J., Newall, A., Denney-Wilson, E., Zwar, N., . . Harris, M. F. (2012). Which providers can bridge the health literacy gap in lifestyle risk factor modification education?: A systematic review and narrative synthesis. BMC Fam Pract, 13, 44. https://doi.org/10.1186/1471-2296-13-44

Griffey, R. T., Kennedy, S. K., D'Agostino McGowan, L., Goodman, M., \& Kaphingst, K. A. (2014). Is low health literacy associated with increased emergency department utilization and recidivism? Acad Emerg Med, 21(10), 1109-1115. https://doi.org/10.1111/acem.12476

Kutner, M., Greenberg, E., Jin, Y., \& Paulsen, C. (2006). The health literacy of America's adults: Results from the 2003 National Assessment of Adult Literacy. Retrieved from http://nces.ed.gov/pubsearch/pubsinfo.asp?pubid=2006483

Literacy for Life. (2016). About Literacy for Life. Retrieved from https://literacyforlife.org/about/ Literacy for Life. (2016). HEAL Program. Retrieved from https://iteracyforlife.org/programs/

McDonald, J. H. (2014). Handbook of Biological Statistics (3rd ed. Vol. 2018). Baltimore, Maryland: Sparky House Publishing.

Mellor, J., \& Uselton, E. (2016). HEAL Program Evaluation Report, 2014-2015. Retrieved from

Milwaukee Health Department. MKE Elevate: A community health improvement plan. Retrieved from https://city.milwaukee.gov/health/MKE-Elevate.htm\#.W_NwSehKhPY 
Office of Disease Prevention and Health Promotion. (2019). Health Communication and Health Information Technology. Retrieved from https://www.healthypeople.gov/2020/topicsobjectives/topic/health-communication-and-health-information-technology/objectives

Ratzan, S., \& Parker, R. (2000). Introduction. Retrieved from Bethesda, MD:

Taggart, J., Williams, A., Dennis, S., Newall, A., Shortus, T., Zwar, N., . . Harris, M. F. (2012). A systematic review of interventions in primary care to improve health literacy for chronic disease behavioral risk factors. BMC Fam Pract, 13, 49. https://doi.org/10.1186/1471-2296-1349

U.S. Department of Health and Human Services. (2010). National action plan to improve health literacy. Retrieved from https://health.gov/communication/initiatives/health-literacy-actionplan.asp 


\section{Author Information}

*Abiola O. Keller

College of Nursing

Marquette University

Clark Hall 363

PO Box 1881

Milwaukee WI 53201-1881

Telephone: 414-288-8569

Email: Abiola.keller@marquette.edu

Abiola O. Keller, PA-C, MPH, PhD is an Assistant Professor in the College of Nursing at Marquette University. Her research focuses on depression, medication adherence, and chronic disease self-management with particular interests in health disparities. In particular, her work emphasizes partnering with community agencies and using Community Engaged Research methodologies to address self and symptom management.

Amy Vuyk

Curative Care Network

amymaulbetsch@gmail.com

Telephone: 414-526-8575

Amy Vuyk, MS, CCC-SLP is a pediatric speech language pathologist at Curative Care Network in Mílwaukee, W1. Amy earned a B.S. in Education from the University of Wisconsin-Madison in 1993. She completed an MS in Speech and Language Pathology at Marquette University in 1995.

Joshua Knox

Department of Physician Assistant Studies

Marquette University

1700 West Wells Street

Milwaukee WI 53233

Email: joshua.knox@marquette.edu

Telephone: 414-288-8483

Joshua Knox, PA-C, MA is a Clinical Associate Professor in the Department of Physician Assistant Studies at Marquette Univerisity and Associate Director of the Marquette University Emergency Medicine Post-graduate Physician Assistant Program. His areas of clinical expertise and research interests include gastroenterology, hematology/oncology, emergency medicine, public health, and health literacy.

*Corresponding author 